\title{
An ensemble feature selection approach using hybrid kernel based SVM for network intrusion detection system
}

\author{
Gaddam Venu Gopal ${ }^{1}$, Gatram Rama Mohan Babu² \\ ${ }^{1}$ Y.S. Rajasekhar Reddy University College of Engineering \& Technology, Acharya Nagarjuna University, Nagarjuna \\ Nagar, Guntur, India \\ ${ }^{2}$ Department of Information Technology, RVR \& JC College of Engineering, Chowdavaram, Guntur, India
}

\begin{tabular}{l} 
Article Info \\
\hline Article history: \\
Received Jul 18, 2020 \\
Revised Apr 29, 2021 \\
Accepted May 4, 2021 \\
\hline
\end{tabular}

\section{Keywords:}

Feature selection Hybrid kernel Intrusion detection system Kyoto $2006+$

Support vector machine

\begin{abstract}
Feature selection is a process of identifying relevant feature subset that leads to the machine learning algorithm in a well-defined manner. In this paper, a novel ensemble feature selection approach that comprises of relief attribute evaluation and hybrid kernel-based support vector machine (HK-SVM) approach is proposed as a feature selection method for network intrusion detection system (NIDS). A Hybrid approach along with the combination of gaussian and polynomial methods is used as a kernel for support vector machine (SVM). The key issue is to select a feature subset that yields good accuracy at a minimal computational cost. The proposed approach is implemented and compared with classical SVM and simple kernel. Kyoto $2006+$, a benchmark intrusion detection dataset, is used for experimental evaluation and then observations are drawn.
\end{abstract}

This is an open access article under the $\underline{C C B Y-S A}$ license.

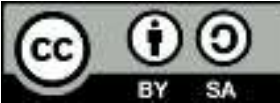

Corresponding Author:

Gaddam Venu Gopal

Dr. Y.S. Rajasekhar Reddy University College of Engineering \& Technology

Acharya Nagarjuna University

Nagarjuna Nagar, Guntur, India

Email: venugopal.gaddam@gmail.com

\section{INTRODUCTION}

Now-a-days, network intrusion detection system plays an important role. Owing to the rapid growth in the use of web usage and other network services, these network services are prone to vulnerable and need to be provided with more security. Network intrusion detection system (NIDS) is one of the solutions that identifies and prevents the intruder from penetrating into the network and from doing any malicious activities.

For the last few decades, most of the researchers are working in the field of NIDS and proposing and implementing their work on different benchmark datasets available. As these datasets are very big in size and provided ith diversified high dimensionality, most of the authors have proposed machine learning techniques not only for attack detection but also quick detection of intruder. Feature selection and feature reduction are some of the methods to choose subset selection of dimensions. Dimensionality reduction helps the NIDS in increasing the detection rates by eliminating those irrelevant features and also minimizes the cost of the detection system.

Several machine learning techniques like decision trees, ada boosting, random forest, artificial neural networks, and SVM, are used as classifiers for predicting whether the request is an attack or a legitimate one. Among these classifiers, SVM is one of the prominent machine learning techniques that is used especially, if the problem domain is a binary classifier. Support vector machine (SVM) [1] is a 
statistical learning based linear classifier introduced by Vapnik and team in 1990s. In order to solve the quadratic optimization problem, SVM algorithm maximizes the margin among the training data through linearly separable hyperplane.

A hyperplane in a high dimensional space has the largest distance to the nearest training data points of any class which is known as the optimum separation hyperplane. SVM algorithm, along with non-linear characteristics improves the ability of generalization, solves the high-dimensional problems, detection rate and also provides a better solution for fault detection and prediction [2]. A kernel function is used in linear classifiers to solve non-linear problems.

In SVM classification algorithm, kernel plays an important role that can be applied in SVM to convert original input space i.e., a high dimension space into a nonlinear mapping. The primary goal of any intrusion detection system is to identify attacks with highest detection rates. Apart from the detection rate, another requirement is quick detection i.e., minimization of the computational time is also important in NIDS [3], [4]. In the network environment, servers need to provide services with quick response to its clients. In this view, NIDS having high computational time, can not be adopted as a detection system for the servers.

To fulfil these goals a feature selection method namely, relief feature selection model is used and a hybried kernel based SVM classifier (HKSVM) is adopted as a classifier. The objective of the proposed work is to develop an intrusion detection system [5], [6] using an ensemble approach along with relief feature estimator and HK-SVM to gain high accuracy and good computational times.

The remaining paper is organized as stated. Section 2 gives a brief review of related work in the field of NIDS and SVM. Basics and dataset description is presented in Section 3. The proposed methodology is elaborately discussed in Section 4 and in Section 5 results. Finally, in Section 6, conclusions are derived.

\section{RELATED WORK}

For the past few decades, several researchers are working in the field of NIDS and also in SVM. There are developments in SVM as well as NIDS. This section discusses the related research work done by various authors in the area of NIDS using SVM [7]-[9]. Table 1 presents a brief description of various researchers along with the datasets are considered, and methodologies are adopted/proposed.

Table 1. Related work

\begin{tabular}{ll}
\hline $\begin{array}{l}\text { Ref. } \\
\text { No. }\end{array}$ & Description \\
\hline [7] & $\begin{array}{l}\text { The authors of this paper proposed a methodology to improve the performance of the SVM using fusion of the genetic } \\
\text { algorithm for the SVM. KDD } 1999 \text { dataset that is used to test their accuracy. } \\
\text { The authors of this paper suggested a hybrid genetic algorithm for SVR as its kernel function. } \\
\text { The model was tested on temperature and load datasets and compared with different kernel methods. } \\
\text { [10] } \\
\text { The authors of this paper presents a mutual information gain based feature selection method for selecting feature subset and } \\
\text { tested using LS-SVM classifer on the KDD1999 dataset. Accuracy, FP rate and other measures were considered. } \\
\text { The authors of this paper presents a detailed study which is provided on various feature selection algorithms in the field of } \\
\text { intrusion detectin system. PCA, Correlation coefficient, and Fusion of Genetic Algorithm, were presented and tested on KDD } \\
\text { 1999 dataset. Detection rate and computational time were considered to compare the models. } \\
\text { The authors investigated the performance of two classification algorithms namely SVM and Artificial Neural Networks (ANN). } \\
\text { Three parameters SVMs train, and run, an order of magnitude faster were considered and concluded that SVM is better than } \\
\text { ANN for NIDS. } \\
\text { The authors of this paper studied NIDS datasets KYOTO 2006+. A Decision Tree algorithm (J48) was applied on this datasets } \\
\text { and gained about 97\% of accuracy }\end{array}$ \\
[14]
\end{tabular}

Most of the works done by the researchers concentrated on a single kernel based SVM and feature selection algorithms which are also concentrating only on the detection rates. Very few authors have concentrated on the computational time which is very important for time critical problems like NIDS. The motivation of the paper is to develop an ensemble classifier using Relief feature estimator and a hybrid kernel based SVM for feature selection process that gives a good accuracy with the best computational time as well.

\section{RESEARCH METHOD}

This section presents the proposed methodology by providing the detailed description of the dataset, basic notations and concepts used, relief algorithm and finally, various performance metrics.

\subsection{Proposed methodology}

The proposed methodology consists of three phases. They are data preprocessing phase, Feature Selection, Evaluation phase and result analysis phase. Figure 1 presents the proposed methodology. In the 
data preprocessing phase, KYOTO 2006+ dataset is preprocessed. The preprocessing consists of two parts namely, i) data transformation and ii) normalization.

Since SVM is a distance based classifier, it works on numeric data whereas KYOTO 2006+ dataset consists of non-numeric features. These feature values are transformed into numeric values. To avoid feature range influence on distance measure and other classification process, each feature has undergone normalization. The normalization technique that is applied in this experiment is min-max normalization. The formula is given (1),

$$
x^{\prime}=\left(\frac{x-\min _{X}}{\max _{X}-\min _{X}} \times\left(n \_\max _{X}-n \_\min _{X}\right)+n \_\min _{X}\right)
$$

Where $\mathrm{x}$ and $x^{\prime}$ are actual and transformed values of feature vector respectively. $\min _{X}$, and $\max _{X}$ are minimum and maximum values of feature $\mathrm{x}$ and $n \_\min _{X}$ and $n_{-} \max _{X}$ are new minimum and new maximum values of the range for which the feature is to be normalized. After the completion of the preprocessing phase, the resultant data is stored as KYOTO-Norm that is supplied as an input for the Feature selection phase.

In feature selection phase, a novel ensemble feature elimination method is proposed on Kyoto-norm dataset. The feature selection approach is given as follows:

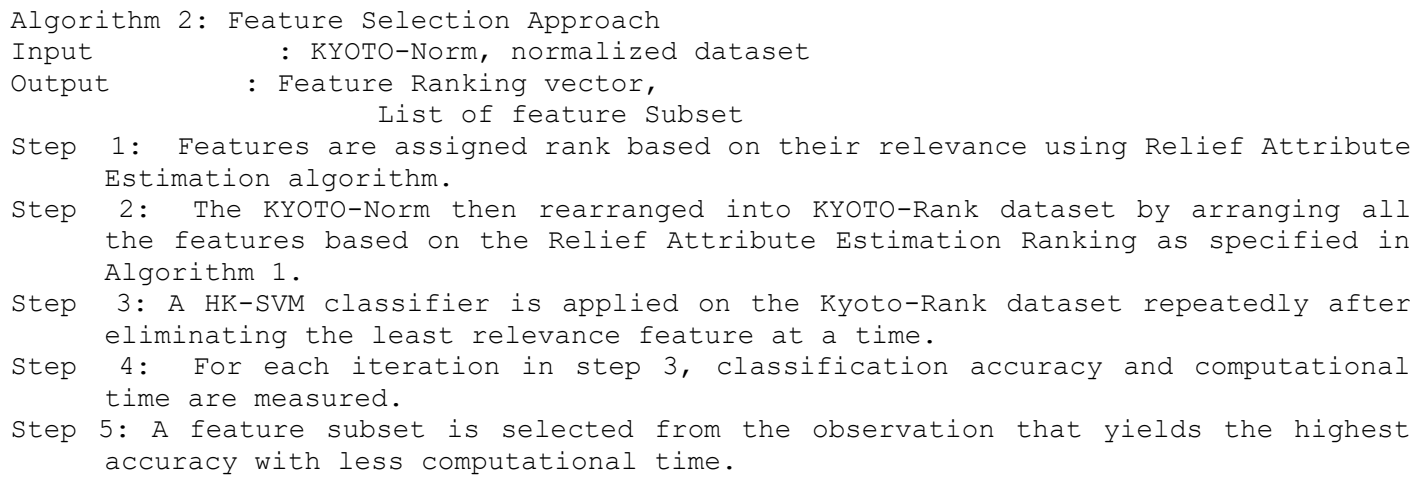

Finally, the results will be compared with the traditional SVM method.

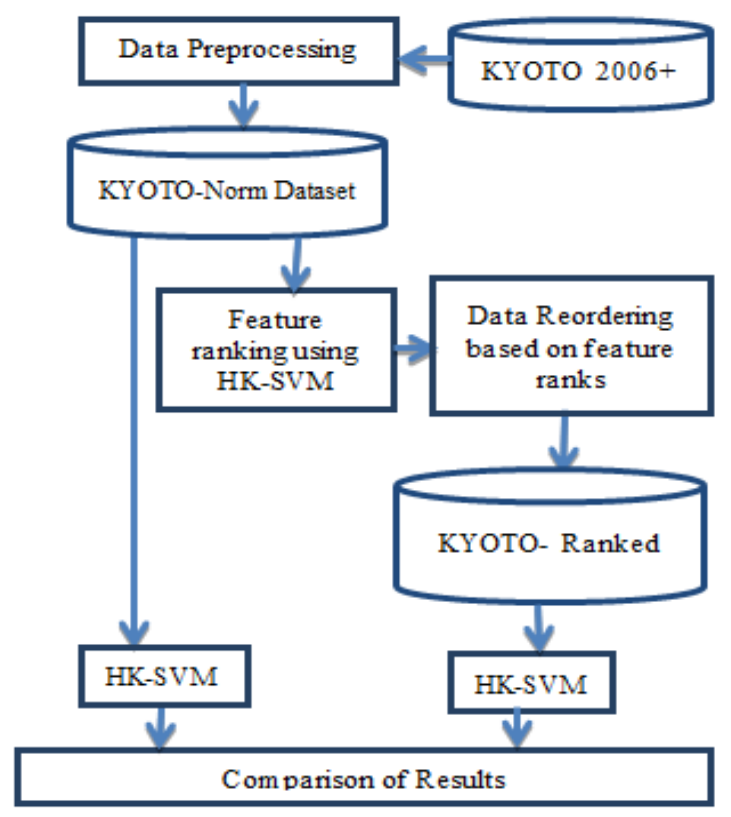

Figure 1. Proposed methodology FSHK-SVM 


\subsection{Dataset description}

Kyoto 2006+, a benchmark dataset is used in this process. The dataset used in the proposed work is a real network traffic dataset that is known as Kyoto 2006+. It is helpful to implement intrusion detection methods. This dataset is collected from honeypots. The dataset was captured from November, 2006 to August, 2009. One of the advantages of Kyoto 2006+ dataset consists of recent trends of attacks that are generated with the help of honeypots. Kyoto dataset is available with 24 features, out of them 14 are derived from KDDCUP'99 dataset [15]-[17], and 10 more features are added that may be helpful in detecting the kind of attacks very effectively in the network.

In the proposed methodology, implementation takes place only one day data set i.e., on $5^{\text {th }}$ August, 2009 consisting of 1,28,347 samples and 24 features out of which, 18 input features are selected because three features namely, IDS_detection, Malaware_detection and Ashula_detection are all considered as prediction features in support of class label [18]. Two more features are IP addresses for both source and destination that have extremely large range of values. Owing to the above reasons, these features are removed for the experiment. The resultant 18 input features that are used in this approach are listed in Table 2.

The class label of this dataset is a three valued feature that defines whether the sample is an attack or a normal request. Those three values are $\{-2,-1,1\}$ to represent whether the request is an unknown attack, known attack or a normal respectively. The percentage of unknown attack samples is less i.e., about $0.7 \%$, as it is difficult to predict unknown attacks. In order to make it simplified, binary classification is followed as known and unknown samples [19], [20]. The data set selected for this study is from the day $5^{\text {th }}$ of the August 2009, Kyoto 2006+ dataset.

Table 2. Selevted features from kyoto 2006+ dataset

\begin{tabular}{ll}
\hline Feature \# & \multicolumn{1}{c}{ Feature Name } \\
\hline Feature1 & Duration \\
Feature2 & Service \\
Feature3 & source_bytes \\
Feature4 & destination_bytes \\
Feature5 & Count \\
Feature6 & same_srv_rate \\
Feature7 & serror_rate \\
Feature8 & srv_serror_rate \\
Feature9 & dst_host_count \\
Feature10 & dst_host_srv_count \\
Feature11 & dst_host_same_src_port_rate \\
Feature12 & dst_host_serror_rate \\
Feature13 & dst_host_srv_serror_rate \\
Feature14 & Flag \\
Feature15 & Label \\
Feature16 & source_port_number \\
Feature17 & destination_port_number \\
Feature18 & Duration1 \\
\hline
\end{tabular}

\subsection{Relief algorithm}

RELIEF is an efficient feature ranking model evaluated based on the contextual information. Relief algorithm estimates feature quality for a specific task by computing dependencies among features. The basic idea of RELIEF approach is to estimate the quality of features according to how well their values distinguish between instances that are near to each other [21], [22].

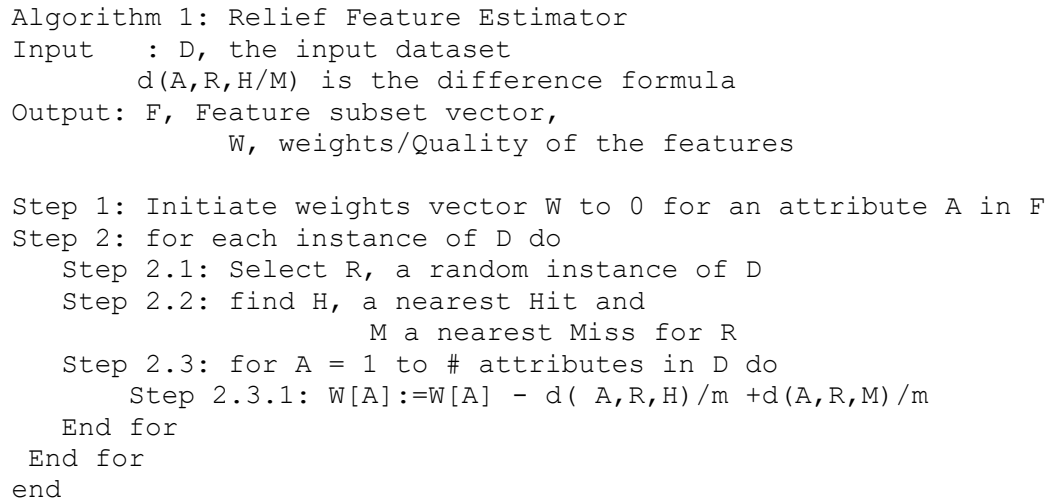


The algorithm works like this, let a training dataset be $\mathrm{D}$ and $\mathrm{F}$ is the feature space of $\mathrm{D}$. A random instance $\mathrm{R}$ from $\mathrm{D}$ is selected, then it picks up two nearest neighbors $\mathrm{H}$ nearest Hit and $\mathrm{M}$ nearest Miss, where $\mathrm{H}$ is from the same class as $\mathrm{R}$ and $\mathrm{M}$ is from other class. Now, the difference between these two classes are computed and added to the Weight Vector for each attribute of A. The difference formula is computed as follows:

$$
\mathrm{d}\left(\mathrm{A}, \mathrm{I}_{1}, \mathrm{I}_{2}\right)=\frac{\mathrm{V}\left(\mathrm{A}, I_{1}\right)-\mathrm{V}\left(\mathrm{A}, I_{2}\right)}{\max (A)-\min (A)}
$$

where $I_{1}, I_{2}$ are two instances, and $V$ is the value of an instance at attribute $A, \max (A), \min (A)$ are the maximum and minimum values of the attribute $\mathrm{A}$.

\subsection{Kernel approaches:}

The accuracy of any SVM depends on the selected kernel and parameters provided to it. The hybrid kernel function that is proposed in this article produces good results. The hybrid kernel function of SVM algorithm is applied on a benchmark intrusion detection dataset and then the experiment is executed. The experiment results show that the proposed prediction method has better accuracy when compared with traditional SVM kernel approaches [23], [24].

\section{A. Gaussian kernel}

An exponential decay function which is known as Gaussian kernel, is computed between a data point and each of the support vectors. It is similar to a weighted linear kernel. The maximum value of a Gaussian function is attained at the support vector that is uniformly decayed among all directions.

$$
k_{1}(x, y)=\exp \left(\frac{-\|x-\dot{x}\|^{2}}{2 \sigma^{2}}\right) \text { for } \sigma>0
$$

B. Polynomial Kernel

Polynomial kernels are commonly used with support vector machines that specify the similarity of feature vectors in the dataset over polynomials of the original features. In a Polynomial kernel, $\mathrm{K}_{2}$ represents Feature Space's inner product F [25]:

$$
k_{2}(x, y)=\left(x^{T} y+C\right)^{d}
$$

where $\mathrm{x}$ and $\mathrm{y}$ are inputs in the sample space, $\mathrm{d}$ is degree of the polynomial.

The proposed hybrid kernel $\mathrm{H}(\mathrm{x}, \mathrm{y})$ is derived from the summation of (1) and (2) as:

$$
H(x, y)=k_{1}(x, y)+k_{2}(x, y)
$$

The following are algorithms for the proposed Hybrid Kernel based SVM for Intrusion Detection System. Algorithm 1 represents the classification approach and Algorithm 2 presents the proposed Hybrid Kernel function.

Let the training sample set $\mathrm{S}=\left\{\left(\mathrm{x}_{1}, \mathrm{y}_{1}\right),\left(\mathrm{x}_{2}, \mathrm{y}_{2}\right),\left(\mathrm{x}_{3}, \mathrm{y}_{3}\right) \ldots \ldots\right\}$, where $\mathrm{x}_{\mathrm{i}} \in \mathrm{R}$ and its respective multiclass labels $\mathrm{y}_{\mathrm{i}} \in\{-2,-1,1\}$. A non-linear mapping $\phi$ from original data to a high-dimensional feature space, therefore, it can be replaced with sample points $x_{i}$ and $x_{j}$ with their mapping $\phi\left(x_{i}\right)$ and $\phi\left(x_{j}\right)$ respectively[13].

$\mathrm{K}$ is the kernel function that $\square \mathrm{x}_{\mathrm{i}}, \mathrm{x}_{\mathrm{j}} \in \mathrm{R}$, satisfies the following equation:

$$
k(x, y)=\{\varnothing(x), \emptyset(y)\}
$$

where $\phi$ is a mapping from $\mathrm{R}$ to a feature space $\mathrm{F}$,

$$
\emptyset: x \rightarrow \emptyset(x) \in F
$$

Hybrid kernel functions has excellent learning capability. In the design of any effective model, there are two essential parameters that influence SVM [26]. Among these parameters, the first parameter C is known as regularization parameter which defines the adjustment cost. This adjustment cost can be computed as the cost of minimization of training error and model complexity; and the second parameter is sigma that considers the non-linear mapping from the available input-space to the high-dimensional feature-space. The present work states additive function of both the Gaussian and polynomial kernels as a hybrid kernel function. 


\subsection{Performance metrics}

Accuracy and computational time are considered as the performance measures for identifying the impact of feature subset on classifiers efficiency. A confusion matrix is calculated with the entries of true positive (TP), true negative (TN), false positive (FP) and false negative (FN) values. Where TP is the total number of correctly predicted normal samples, TN is the total number of correctly predicted attack samples, FP and FN the number of normal samples are predicted as attacks and number of attack samples predicted as normal respectively.

Accuracy is considered to be ratio of total number of testing samples correctly classified out of the total number of samples;

$$
\text { ACCURACY }=\frac{\mathrm{TP}+\mathrm{TN}}{\mathrm{TP}+\mathrm{TN}+\mathrm{FP}+\mathrm{FN}}
$$

\section{RESULTS AND DISCUSSION}

The proposed model is developed in Java 1.7 on Intel core i5 processor, with 4 GB RAM and with Windows 7 environment. From the Kyoto $2006+, 70 \%$ is considered for the training set and $30 \%$ is considered as the test set. Figure 2 and Figure 3 present the computational time and accuracies of the EHKSVM classifier respectively, after each feature elimination after relief feature estimator. Table 3 gives accuries and computational times of two featue subsets with 11 features and 9 features and compared with the HK-SVM classifier with total number of featues. In Figure 2 and Figure 3, It is observed that the classifier EHK-SVM(11) is an ensemble Hybrid SVM classifier with 11 features and EHK-SVM(9) with 9 featuers for both gain in computational time and accuracy are suggested.

Table 3. Accuracy and computational time of the classifiers number of features in parenthesis

\begin{tabular}{ccc}
\hline Algorithm & Accuracy & Time in milliseconds \\
\hline HK-SVM (18) & 92.51 & 27520 \\
EHK-SVM(11) & 99.08 & 17562 \\
EHK-SVM(9) & 92.48 & 15288 \\
\hline
\end{tabular}

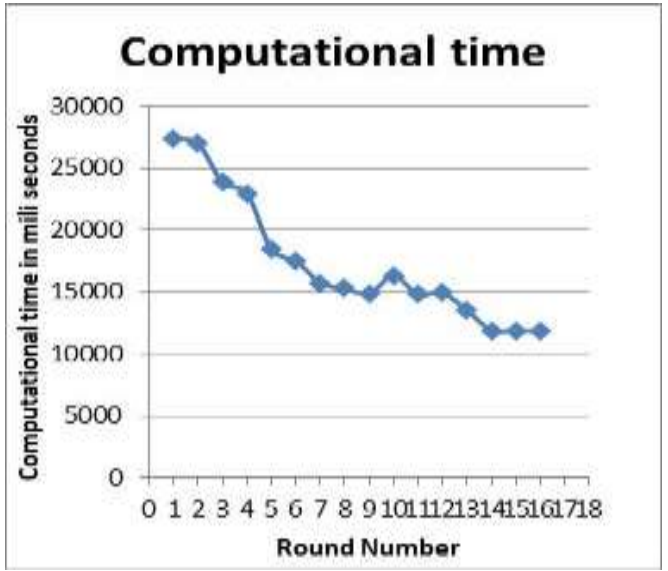

Figure 2. Computational time of the EHKSVM for each iteration

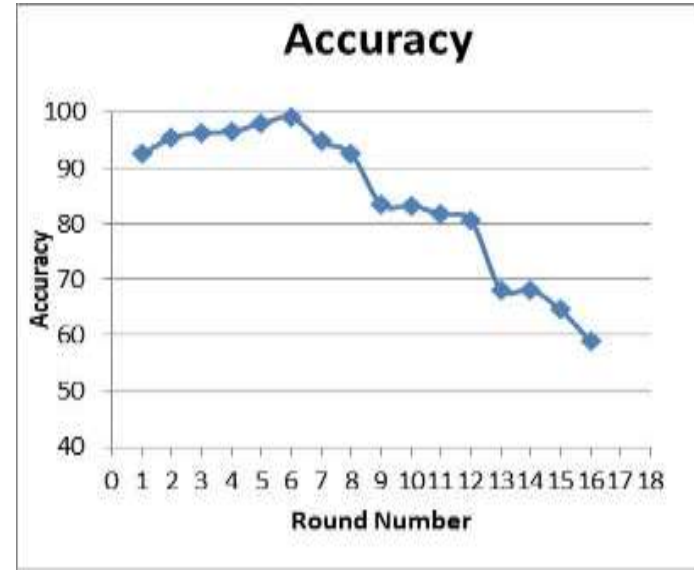

Figure 3. Accuracies of the EHKSVM for each iteration

In Figure 4 and Figure 5, it is observed that the classifier EHK-SVM(11) is exhibiting highest accuracy i.e., $99.02 \%$ at 11 features. The computational time of the classifier is also less when compared to HKSVM with a total number of 18 features. 


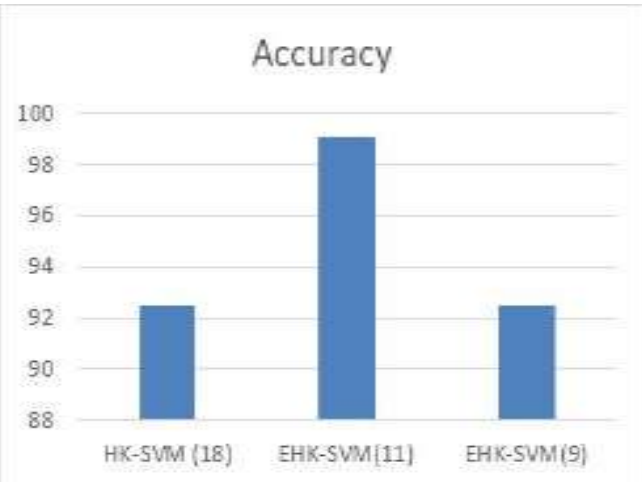

Figure 4. Accuracies of HKSVM, EnsembledSVM with 11 and 9 features

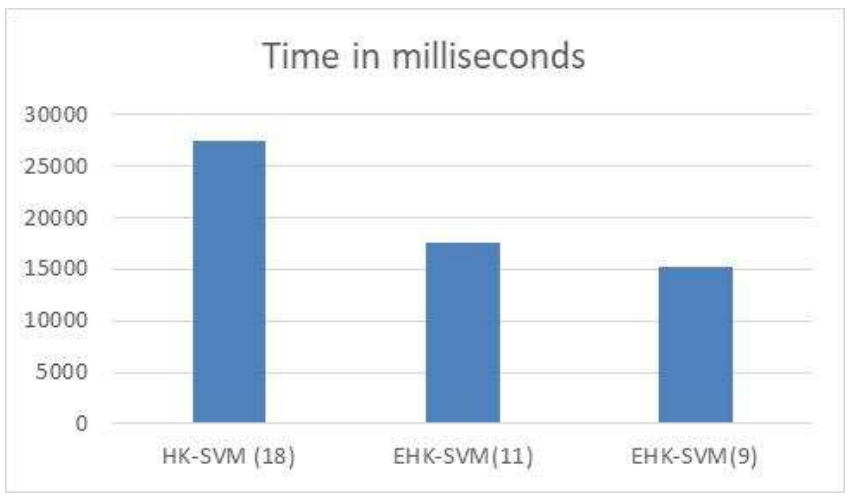

Figure 5. Compurational time of HKSVM, EnsembledSVM (with 11 and 9) in milliSeconds

From the above results the following observations were drawn.

a) A HKSVM classifier with is a hybrid classifier that yields good results than the traditional SVM and SVM with kernel based classifiers is implemented along with Ensembled classifier with feature reduction approach.

b) EHK-SVM(9) is also exhibiting good detection rate i.e., $92.48 \%$ almost as same as the accuracy of HKSVM $(0.03 \%$ difference in accuracies) with more gain in computational time.

\section{CONCLUSION}

In this paper, an ensemble hybrid kernel based SVM is used as a feature selection approach that is implemented and tested on a bench mark dataset, KYOTO 2006+. The proposed method suggests 2 scenarios, one with 11 features i.e., EHK-SVM(11) and another with 9 features i.e., EHK-SVM(9). It is observed that 11-feature scenario is giving highest accuracy which is a good scenario for a requirement of high detection rate whereas the 9-feature scenario gives a good gain computational time that is helpful in quick detection rate. The proposed EHK-SVM is compared to the existing models that uses the same dataset for evaluation and concluded the proposed approach exhibits better accuracy. Owing to these results, the proposed model can be used to implement in real-time environment of NIDS. Implementation of the EHKSVM on Real time dataset generated on NIDS is the future work of this paper.

\section{REFERENCES}

[1] V. N. Vapnik, "Statistical learning theory," vol. 2, 1998.

[2] Avci, Engin, "Selecting of the optimal feature subset and kernel parameters in digital modulation classification by using hybrid genetic algorithm-support vector machines: HGASVM," Expert Systems with Applications, vol. 36, no. 2, 2009, pp. 1391-1402, doi: 10.1016/j.eswa.2007.11.014.

[3] "Nsl-kdd data set for network-based intrusion detection systems." Available on: http://nsl.cs.unb.ca/KDD/NSLKDD.html, March 2009.

[4] B. B Rao, K. Swathi, "Fast kNN classifiers for network intrusion detection system," Indian Journal of Science and Technology, vol. 10, no. 14, pp. 1-10, 2017, doi: 10.17485/ijst/2017/v10i14/93690.

[5] Zhang Qinglei, and Wenying Feng, "Network intrusion detection by support vectors and ant colony," Proceedings. The 2009 International Workshop on Information Security and Application (IWISA 2009). Academy Publisher, 2009, pp. 639-642.

[6] Gu, Chunhua, and Xueqin Zhang, "A rough set and SVM based intrusion detection classifier," 2009 Second International Workshop on Computer Science and Engineering, vol. 2. IEEE, 2009, doi: 10.1109/WCSE.2009.776.

[7] Kim, Dong Seong, Ha-Nam Nguyen, and Jong Sou Park, "Genetic algorithm to improve SVM based network intrusion detection system," 19th International Conference on Advanced Information Networking and Applications (AINA'05), vol. 1 (AINA papers). vol. 2. IEEE, 2005, doi: 10.1109/AINA.2005.191.

[8] Mo, Yuanbin, and Shuihua Xu., "Application of SVM based on hybrid kernel function in heart disease diagnoses," 2010 International conference on intelligent computing and cognitive informatics, IEEE, 2010, doi: 10.1109/ICICCI.2010.96.

[9] Mukkamala, Srinivas, and Andrew H. Sung, "Feature selection for intrusion detection with neural networks and support vector machines," Transportation Research Record: Journal of Transportation Research Record, vol. 1822, no. 1, pp. 33-39, 2003, doi: 10.3141/1822-05. 
[10] Wu Chih-Hung, Gwo-Hshiung Tzeng, and Rong-Ho Lin, "A Novel hybrid genetic algorithm for kernel function and parameter optimization in support vector regression," Expert Systems with Applications, vol. 36, no. 3, 2009, pp. 4725-4735, doi: 10.1016/j.eswa.2008.06.046.

[11] Amiri, Fatemeh, Mohammad Mahdi Rezaei Yousefi, Caro Lucas, Azadeh Shakery, NasserYazdani, "Mutual information-based feature selection for intrusion detection systems," Journal of Network and Computer Applications, vol. 34, no. 4, pp. 1184-1199, 2011, doi: 10.1016/j.jnca.2011.01.002.

[12] Chen You, Yang Li, Xue-Qi Cheng, Li Guo, "Survey and taxonomy of feature selection algorithms in intrusion detection system," International Conference on Information Security and Cryptology, Springer, Berlin, Heidelberg, vol. 4318, 2006, doi: 10.1007/11937807_13.

[13] B. Schölkopf, et. al. "Input space vs. feature space in kernel-based methods," IEEE Trans. Neural Networks, vol. 10, no. 5, pp. 1000-1017, 1999, doi: 10.1109/72.788641.

[14] Sun, Yuxuan, Xiaojun Lou, and Bisai Bao, "A novel relief feature selection algorithm based on mean-variance model," Journal of Information and Computational Science, vol. 8, no. 16, pp. 3921-3929, 2011, doi: 10.24297/ijct.v11i8.7047.

[15] Wang, Ping An, Xu Sheng Gan, and Wen Ming Gao, "Research on Nonlinear modeling Method of Support Vector Machine with Wavelet Derivation Kernel Function," Applied Mechanics and Materials, vol. 687, pp. 1408-1411, Trans Tech Publications Ltd, 2014, doi: 10.4028/www.scientific.net/AMM.687-691.1408.

[16] Cheng Lili, Jianpei Zhang, Jing Yang, Jun Ma, "An improved hierarchical multi-class support vector machine with binary tree architecture," In 2008 International Conference on Internet Computing in Science and Engineering, pp. 106-109, IEEE, 2008, doi: 10.1109/ICICSE.2008.9.

[17] Sun, Yijun, "Iterative RELIEF for feature weighting: algorithms, theories, and applications," IEEE transactions on pattern analysis and machine intelligence, vol. 29, no. 6, pp. 1035-1051, 2007, doi: 10.1109/TPAMI.2007.1093.

[18] Yili Ren, Fuxiang Hu, Hongping Miao, "The optimization of kernel function and its parameters for SVM in welllogging," 13th International Conference on Service Systems and Service Management (ICSSSM), 2016, doi: 10.1109/ICSSSM.2016.7538563.

[19] B. Schölkopf, A. Smola, "Learning With Kernels," Cambridge, MA: MIT Press, 2002.

[20] Chih-Cheng Yang, Wan-Jui Lee, and Shie-Jue Lee, "Learning of Kernel Functions in Support Vector Machines," 2006 International Joint Conference on Neural Networks Sheraton Vancouver Wall Centre Hotel, Vancouver, BC, Canada, 2006, pp. 1150-1155.

[21] Kyoto 2006+ dataset is available on: http://www.takakura.com/Kyoto_data/

[22] Song Jungsuk, et al. "Statistical analysis of honeypot data and building of Kyoto 2006+ dataset for NIDS evaluation," Proceedings of the First Workshop on Building Analysis Datasets and Gathering Experience Returns for Security, ACM, 2011, pp. 29-36, doi: 10.1145/1978672.1978676.

[23] Lu, Yan-Ling, Lei LI, Meng-Meng Zhou, Guo-Liang Tian, "A new fuzzy support vector machine based on the mixed kernel function," In 2009 International Conference on Machine Learning and Cybernetics, vol. 1, 2009, pp. 526-531, IEEE, doi: 10.1109/ICMLC.2009.5212552.

[24] Altun Gulsah, et. al. "Hybrid SVM Kernels for Protein Secondary Structure Prediction," In GrC, pp. 762-765, 2006, doi: 10.1109/GRC.2006.1635912.

[25] A. Bosch, Zisserman A, Munoz X, "Representing shape with a spatial pyramid kernel," Conference on Image and Video Retrieval, 2007, pp. 401-408, doi: 10.1145/1282280.1282340.

[26] S. Boughorbel, J.P. Tarel, N. Boujemaa, "Generalized histogram intersection kernel for image recognition," In IEEE International Conference on Image Processing 2005, Genova, Italy, IEEE, doi: 10.1109/ICIP.2005.1530353.

\section{BIOGRAPHIES OF AUTHORS}

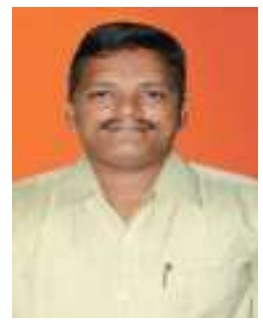

Mr. G. Venugopal, Research Scholar at Acharya Nagarjuna University. Pursuing Ph. D in the field of Machine Learning. As part of his Ph. D work, he is writing this paper. He Completed M. Tech in CSE from Acharya Nagarjuna University (ANU) in the year 2010. He Finished B. Tech in CSE from JNTUH, Hyderabad. His area of interest is Data Mining and Machine Learning. He is a Life member of professional bodies like ISTE, IAENG.

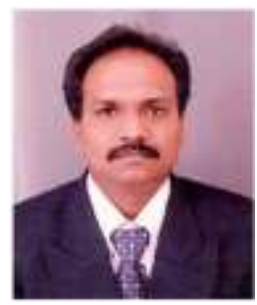

Dr. G. Rama Mohan Babu, received his B.Tech degree in Electronics \& Communications Engineering from Sri Venkateswara University, India. He did his M.Tech in Computer Science \& Engineering from Jawaharlal Nehru Technological University (JNTU), India. He received his Ph.D. from Acharya Nagarjuna University (ANU), India, in Computer Science \& Engineering. $\mathrm{He}$ is currently working as Professor, in the Department of Information Technology at RVR \& JC College of Engineering, Guntur, India. He has more than 19 years of teaching experience. His research areas of interest include image processing, pattern recognition, and data analytics. $\mathrm{He}$ is life member in professional bodies like ISTE and CSI. 